\title{
Resumen
}

La investigación se centra en un análisis acerca de la manera que puede influir un proceso de Due Diligence en la venta de una empresa. En este caso, la venta de una empresa del retail. Se describe la metodología de un Due Diligence, para conocer en profundidad el proceso, se explican los posibles resultados de éste, se identifican el o los efecto(s) en la empresa en estudio y se confirma la real ingerencia del Due Diligence en la venta de la empresa retail, a través de la información pública obtenida. Se presenta, finalmente, el resultado del Due Diligence, que proporciona una serie de circunstancias que pueden influir tanto para la empresa vendedora y el inversor, condicionando la valorización de la empresa, para llegar a un precio final, a una oferta concreta que sea aceptada por ambas partes y que sea justa para cada una de ellas.

Palabras claves: Due Diligence, Retail, Empresa Vendedora, Inversor, Valorización.

\section{ANÁLISIS DE LA INFLUENCIA DE UN DUE DILIGENCE EN LA VENTA DE UNA EMPRESA.}

\section{ANALYSIS OF THE INFLUENCE OF A DUE DILIGENCE IN THE SALE OF A BUSINESS.}

Paulina Alarcón Godoy ${ }^{1}$

\section{Abstract}

This investigation focuses on an analysis about how a process of Due Diligence can influence in the sale of a business. In this case, the sale of a retail company. The methodology of a Due Diligence, in order to know profoundly this process, is described; its possible results are explained, the effect or effects in the company are identified, and the real interference of the Due Diligence in the sale of a retail company is confirmed through public information obtained. Finally, the result of the Due Diligence is presented. This adjusts a series of circumstances that can influence, both the selling company and the investor, conditioning the company's valuation to reach a final price taking into account a real offer which is accepted by both parties and that is fair to each.

Key Words: Due Diligence, Retail, Selling Company, Investor, Valuation. 


\section{INTRODUCCIÓN.}

En el proceso de fusiones y adquisiciones entre empresas que se comienza a llevar a cabo a través de una serie de procedimientos que como lo explican Asesores Norgestión (2010), éste se inicia después de un período de negociación y aproximación entre los vendedores, los inversores o compradores y, luego de que el inversor muestre un interés real, normalmente reflejado mediante una "Carta de intenciones", en la que se recoge precisamente la intención de adquirir la totalidad 0 una parte de una sociedad que se encuentra en determinadas condiciones. A partir de eso, se abre un proceso denominado "Due Diligence" o "Diligencias Debidas", en virtud del cual el adquirente de una empresa realiza, con explícito consentimiento y asistencia del vendedor, una detallada investigación de diferentes áreas del negocio que se quiere adquirir, a objeto de conocer con mayor profundidad aquello que se desea obtener.

El objeto del proceso es que los profesionales o el profesional encargado de realizarlo sea capaz de determinar, por medio de revisiones detalladas del negocio, si el precio que se ha comprometido a pagar por la totalidad o parte de la empresa es justo (Roca, 2010).Con lo anterior se pueden esbozar una serie de acontecimientos, tales como existencia de pasivos ocultos o de activos ficticios, esto desencadena dificultades que pueden afectar la adquisición de una empresa y más que todo a la venta de una empresa, ya sea, disminuyendo el precio pactado, desistiendo por parte de la empresa inversora de la compra, o reformulando los términos generales del contrato.

En razón de lo expuesto, conocer el proceso de "Due diligence" es sumamente importante, ya que de él depende que las intenciones se transformen en un desembolso real y, por lo tanto, en una inversión.

Un buen asesor de "Due Diligence" estará en una posición única para jugar un papel clave, llevando la transacción a un resultado satisfactorio para el comprador y, por ende, para el vendedor (Papa, 2011). Pero éste no siempre es el resultado, por lo que la presente investigación conceptualiza la metodología de un proceso de Due Diligence y la influencia de este proceso en la venta de una empresa. En este caso específico, la venta de una empresa retail. 


\section{DUE DILIGENCE.}

La Due Diligence 0 también denominada Auditoría de Compra, conceptualmente, es un proceso de investigación y análisis cuya finalidad es conocer la totalidad del negocio, ya sea financiera, tecnológica, organizativa y jurídicamente, con objeto de proporcionar información relevante que guíe y soporte la decisión de la empresa compradora. (Restrepo, 2008)

El proceso de investigación del Due Diligence está orientado a identificar preventivamente riesgos patrimoniales, regulatorios y restricciones de diversa índole, que puedan impactar a la compañía $y$, por lo tanto a la proyectada transacción. (Papa, 2011)

El Due Diligence es una práctica común de las personas al momento de embarcarse en una adquisición. A partir de lo señalado, se puede concluir que el objetivo básico de un trabajo de Due Diligence 0 Diligencias Debidas es poder auxiliar al comprador, mediante la discusión de los riesgos y oportunidades que presenta la transacción que se está considerando realizar. A partir de esto se efectúa un análisis basado en la información que facilita tanto el comprador como el vendedor. (Chajón, 2003)

\section{PROPÓSITO DE UN DUE DILIGENCE.}

La revisión legal, financiera, tecnológica, administrativa, organizativa y, en general, integral de empresas, es una práctica recomendable en los casos de operaciones de adquisición de la totalidad, o de parte, del capital social y accionario de una sociedad, por otra interesada en su compra. (Iriarte, San Sebastián, y Sainz, 2010). Esta denominada auditoría de compra, le permitirá saber al inversor qué es lo que compra y cuánto vale realmente la empresa en venta, es decir:
Según Roca (2010), el fin del proceso de Due Diligence es determinar las cualidades del negocio a adquirir, la conveniencia de su adquisición y el precio justo a pagar.

Además, Restrepo (2008), agrega que los principales objetivos es decidir si la transacción encaja con la estratégia que se tiene planeada $y$, asegurar que el precio pagado por el nuevo negocio 0 las condiciones de éste sean razonables, para obtener un retorno adecuado en la inversión. Por su parte, Finanzas Tour (2011), resume las finalidades del proceso, que desde el punto de vista tradicional, permite, entre otros:

- Identificar riesgos

- Servir de soporte en la determinación del valor de la empresa o negocio a adquirir.

- Obtener información valiosa en el proceso de negociación.

- Determinar potenciales sinergias y medidas que mejoren el funcionamiento

- Determinar la estructura de la operación y el mejor método de financiación.

\section{CLASES DE DUE DILIGENCE.}

La extención de la Due Diligence depende de las necesidades del comprador 0 inversionista. Según, Carrera (2011), las modalidades más requeridas son las siguientes:

- Revisión Legal y laboral

- Revisión Operativa

- Revisión Circunstancial

- Revisión Tributaria

- Revisión Financiera

Además Iriarte, San Sebastián, y Sainz, (2010), agregan a las revisiones anteriores otros tres tipos:

- Revisión Medioambiental

- Revisión Comercial 
- Revisión de los Sistemas de Información (IT)

\section{TIPOS DE TRANSACCIONES DE UN DUE DILIGENCE.}

Fusiones y adquisiciones son los procesos más comunes que requieren IIevar a cabo un trabajo de Due Diligence, pero éstas no son las únicas, como lo indica Chajón, (2003), existen diferentes transacciones que se pueden realizar, donde un Due Diligence se puede hacer presente. Algunas de éstas son:

1. Fusiones: Se habla de fusión cuando dos 0 más compañías deciden juntar sus patrimonios para formar una nueva empresa.

\section{a. Fusión por la creación de} una compañía nueva: consiste en la fusión de dos 0 más compañías para la creación de una sociedad, a la cual cada una aporta la totalidad de su patrimonio y produce la disolución anticipada de cada una de éstas.

b. Fusión por absorción: Se da "cuando la fusión resulta de la absorción de una o varias compañías por otra ya existente. Implica la disolución de las compañías absorbidas, las cuales desaparecen después de haber trasferido la totalidad de sus patrimonios a la sociedad absorbente." (Espiñeira, Sheldon y Asociados, 2008, 3)

2. Adquisiciones: "cuando una empresa compra las acciones y/o activos de una compañía, suficiente para tener el control sobre la misma, sin llevar a cabo la fusión de sus patrimonios. El objetivo final es crear valor." (Espiñeira, Sheldon y Asociados, 2008, 3)
3. Disposiciones: Resulta de la venta de una compañía o de un negocio que describe la acción del vendedor mediante una adquisición. (Chajón, 2003)

4. Disoluciones: La disolución es el acto jurídico que abre el proceso de liquidación que dará lugar a la extinción de la sociedad como contrato y como persona jurídica. La disolución en sí no pone fin a la sociedad ni paraliza totalmente su actividad, aunque ésta pasa a ser liquidataria, por lo que se añade a su denominación la expresión en liquidación. (Monclús, 2000)

5. Alianza estratégica: Es la colaboración 0 soporte entre dos 0 más partes sin que formen una nueva entidad. (Chajón, 2003)

\section{FASES DE LA DUE DILIGENCE.}

Según Tarabini-Castellani, (2010) existen fases que normalmente constan en un Due Diligence estas son:

- Fase preliminar: En la que se determina el objeto y alcance del trabajo.

- Fase de Investigación: En la que se recopila toda la información necesaria para el trabajo.

\section{- Fase de revisión de la información:}

En la que se revisa la información aportada por la compañía.

- Fase de revisión de operaciones: Donde se evalúa las operaciones principales de la compañía.

- Fase de revisión del cumplimiento de aspectos legales: En determinado tipo de trabajos se aprovecha la Due Diligence para la redacción de contratos que generen garantías e identifiquen y 
acoten los riesgos de la transacción.

- Fase de emisión de informes: En la que se genera el informe resumen del trabajo realizado y sus conclusiones.

\section{METODOLOGÍA.}

Desde un punto de vista metodológico, la investigación a realizar se hará sobre un análisis de tipo cualitativo y de carácter comprehensivo.

Los criterios de explicación serán las etapas del proceso de Due Diligence, definidos mes a mes por Vélez (2003) estos son:

Se comparan, de las etapas contenidas en la teoría correspondiente al Due Diligence, definidas por Vélez (2003) y lo que ha realizado la empresa en estudio, con la información entregada a través de la SVS y los medios de comunicación escritos.

\section{RESULTADOS.}

A continuación se detallan los resultados obtenidos de las etapas señaladas por Vélez, logrando una comparación de lo realizado por la empresa en estudio y lo que se define metodológicamente en el proceso.

\section{MES 1}

\section{- Identificar asesor}

En agosto de 2011, la compañía en estudio contrata el servicio del Banco de inversiones Altis. Altis es un banco de inversiones, cuyo gerente general es Alejandro Puentes, el gerente de finanzas es Tomás Müller y su gerente de servicios institucionales es José Gabriel Palma. El mismo Sr. Puentes, ingeniero Comercial, con MBA en Harvard, estaría a cargo de la operación. Trabaja con la información financiera de la empresa, para valorizarla y, con ello, iniciar el proceso de búsqueda de posibles interesados en ingresar a su propiedad. El objetivo de la empresa es buscar una fórmula que capitalice a su cadena de retail. Esto a través de la búsqueda de un socio.

(La Segunda, 2011)

\section{- Desarrollar estrategia de venta.}

La estrategia que se consideraría en este caso es que un nuevo socio ingresará a la propiedad de la compañía Retail, esto a través de un aumento de capital, con lo que, lo más probable sea que el socio tome el control de la empresa. (La Estrategia, 2011)

MES 2

- Preparación del cuaderno de ventas

El Banco de inversiones Altis, comienza a analizar una estrategia para la venta, basándose en el tipo de negocio. Esto a partir de que existe otra cadena retail, que también ha aprobado un aumento de capital, pero esa cadena tiene complicaciones judiciales potentes que podrían persistir por muchos años, Io que hace que su venta tenga una mayor ventaja competitiva, respecto de la otra empresa, La Polar S.A. Con lo expresado, Altis comienza a buscar a los mismos interesados en comprar dicha cadena retail, no sin antes dejar de mencionarles que llegarían con esto al mismo sector de mercado. (La Estrategia, 2011) (La Segunda, 2011) 
Cuadro $N^{\circ}$ 1: Relación de las etapas de una negociación

\begin{tabular}{|c|c|c|}
\hline Mes1 & Mes 4 & Mes 7 \\
\hline Identificar al asesor & $\begin{array}{l}\text { Visitas de compradores } \\
\text { potenciales }\end{array}$ & Due Diligence \\
\hline Desarrollar estrategia de venta & $\begin{array}{l}\text { Recepción de ofertas no } \\
\text { vinculantes }\end{array}$ & \\
\hline Mes 2 & Mes 5 & Mes 8 \\
\hline $\begin{array}{c}\text { Preparación del "cuaderno de } \\
\text { ventas" }\end{array}$ & Negociaciones & $\begin{array}{l}\text { Redacción y negociación } \\
\text { de contratos }\end{array}$ \\
\hline $\begin{array}{l}\text { Identificación de compradores } \\
\text { potenciales }\end{array}$ & Ofertas finales & \\
\hline Valoración & Selección del comprador & \\
\hline Mes 3 & Mes 6 & Mes 9 \\
\hline Enviar perfil ciego & $\begin{array}{l}\text { Redactar acuerdo de } \\
\text { intenciones }\end{array}$ & Cierre de la operación \\
\hline $\begin{array}{l}\text { Enviar acuerdo de confiden- } \\
\text { cialidad }\end{array}$ & Due Diligence & \\
\hline
\end{tabular}

Fuente: Vélez,I. 2003

- Identificación de compradores potenciales

Entre los primeros interesados estarían aquellos a los que se les habría ofrecido directamente la posibilidad de ingresar a la propiedad. Entre ellos estarían el holding de los Said, el Grupo Saieh, la familia Hurtado Vicuña y los Luksic. A la lista se le sumarían fondos de inversión extranjeros, que verían potencial de crecimiento en la firma de retail, luego de que hace dos años estuvo al borde de la quiebra. (La Estrategia, 2011)

\section{- Valoración}

Según Vega, (2011), la empresa retail provoca varios interesados, lo que hace que se valorice en 100 millones de dólares, esto porque su cartera es pequeña, pero mucho más sana de lo que se cree, registra un potencial interesante y apunta a un sector de mercado distinto.

\section{MES 3}

- Enviar perfil ciego

A la mayoría de los empresarios les gustaría mantener totalmente confidencial la venta de la empresa, sin embargo, no se puede vender una empresa con la documentación metida en un cajón. Resulta necesario ponerse en contacto con los potenciales compradores y explicarles la oportunidad. Para este fin, los asesores han desarrollado una metodología de aproximación que busca preservar la confidencialidad del proceso, durante la búsqueda de inversores realmente interesados y garantizar que, una vez que se ha develado a estos la identidad de la compañía en venta, se siga manteniendo esta confidencialidad. (ONEtoONE, Asesores financieros, fusiones y adquisiciones)

- Enviar acuerdo de confidencialidad 


\section{MES 4}

- Visitas de compradores potenciales.

\section{- Recepción de ofertas no- vinculantes}

El Banco de inversión Altis, comienza a recibir ofertas de empresas, las que manifestan su interés por comprar y ser el nuevo socio mayoritario de la compañía. Recibe el interés de tres inversionistas, cuyos nombres se mantienen bajo estricta confidencialidad. Hasta ese momento, solo había admitido el interés, $A B C D i n$, compañía que hasta evaluaba la presentación de una oferta. El abogado de los dueños del retailer en venta, Manuel José Vial, comenta, en ese entonces, que son las primeras ofertas, pero que esperan recibir más interesados y que eso sería beneficioso porque habría más competencia. (Ferrando, 2011)

\section{MES 5}

\section{- Negociaciones}

Acerca de las negociaciones, el abogado de la cadena retail aseguraba que "la decisión final la tomarán los actuales socios de la compañía, junto a los bancos, coordinados por Altis" (Vial, 2011). Los bancos deben participar, pues son acreedores muy importantes de la compañía (la firma le adeudaba más de US\$ 300 millones), además, agrega: "todos los inversionistas interesados tendrán que conversar con la banca sobre sus propuestas y sobre las condiciones de funcionamiento futuro de la compañía" (Vial, 2011).

\section{- Ofertas finales}

Detrás de la cadena retail estuvieron $A B C$ Din, el fondo argentino Leadgate y Parque Arauco, pero la oferta más interesante la recibieron en la tarde del jueves 6 de Noviembre, Horst Paulmann, el presidente de Cencosud, analizó la propuesta, la que le interesó por lo que dio el vamos a la operación, la que considera 100 millones de dólares por el $85,58 \%$ de las acciones de la cadena y, además, un asiento como presidente honorario a dueño de la compañía en estudio, aquello en reconocimiento a su trayectoria. (Vega,2011)

\section{- Selección del comprador}

La de Cencosud era la mejor de todas las ofertas. La familia controladora de la empresa, así lo consideró, ya que según la información publicada, valoraron muchísimo el gesto de Paulmann hacia el dueño, el cual se refirió meses antes a que era necesario: "hacer todo lo que se pueda para que la empresa no se liquide" (Calderón, 2011).

\section{MES 6}

- Redactar acuerdo de intenciones

El día 7 de Octubre del año 2011, Cencosud informa a través de un hecho esencial enviado a la SVS, en el que hace referencia a la variada información que ha aparecido en la prensa, dando cuenta de la situación de la sociedad retail en cuestión y el posible ingreso de diferentes empresas y/o grupos económicos del mercado a la propiedad de dicha compañía, siendo Cencosud S.A. una de las empresas nombradas. 
Al respecto, informamos que con esta fecha hemos suscrito una carta de intención con los accionistas de dicha sociedad, con el objetivo de incorporarnos a la propiedad de la misma, a través de un aumento de capital, suscribiendo un $85,58 \%$ del total de las acciones por el monto de $\$ 51.500 .000$. Todo ello, sujeto a ciertas condiciones y términos habituales en estos tipos de operaciones, incluyendo el acuerdo de los documentos definitivos.

\section{- Due Diligence}

De este modo, el 14 de 0ctubre del 2011, a través de un segundo hecho esencial enviado a la SVS, en el que informa que de acuerdo con el hecho esencial enviado el 7 de Octubre, en el que se dio cuenta de la suscripción de la carta de intención con los accionistas de la sociedad retail en estudio, cuyo objetivo es la incorporación a la propiedad de ésta, en los porcentajes ahí indicados, hemos estimado conveniente complementar dicha información de la manera que pasa a expresarse:

- Uno.- La materialización definitiva del acuerdo alcanzado se encuentra sujeta a la verificación de una serie de condiciones, consistentes en hechos que serán materia de revisión a través de un proceso de Due Diligence. Este último, se centrará en aspectos legales, comerciales $\mathrm{y}$, sobre todo, financieros de la compañía, teniendo en especial consideración los límites acordados por las partes como máximos, en materia de asunción de la responsabi- lidad en materia de pasivos financieros, tributarios y otros relevantes, para cada una de ellas y que fueron determinantes al minuto de suscribirse la ya indicada carta de intención;

- Dos.- Las partes han fijado como plazo máximo, para IIevar adelante el proceso de Due Diligence y, en definitiva, materializar la posible operación, 45 días a contar del 7 de 0ctubre de 2011.

\section{MES 7 \\ - Due Diligence}

Tras fijarse los 45 días de plazo para la realización del Due Diligence, el cual, según el hecho esencial enviado a la SVS, se centrará en aspectos legales, financieros y comerciales de la compañía en estudio, se comienza a realizar una investigación paralela por la FNE (Fiscalía Nacional Económica), por lo que comenzaron las preocupaciones y el temor de que el plazo para terminar el Due Diligence y cerrar la operación podía depender de lo que hiciera la Fiscalía. (El Mercurio, 2011) La investigación de la FNE es delicada y se trata de los potenciales riesgos a la competencia. Esta investigación, como se mencionó, nació una vez que se dio a conocer la operación de compra, una vez iniciado el Due Diligence. La preocupación de la entidad Fiscal, no tiene que ver con que Cencosud pasara a ser una compañía dominante en el sector retail, sino más bien por el rol que tendría el dueño de la compañía retail, en estudio, al interior de Cencosud, esto dado que él también forma parte del 
directorio de Ripley, otra de las grandes empresas retail del país, por lo que se puede interpretar otras intenciones dentro de la transacción y pueden existir conflictos de interés al estar relacionado con dos grandes retailers de Chile. Por lo que la principal preocupación sería el manejo de información privilegiada que tendría el dueño de la empresa en estudio, de dos grandes competidores. Durante la investigación del FNE, la familia controladora del retail en estudio comienza a entregar todos los antecedentes solicitados y hacen referencia a los mínimos porcentajes de participación que tendría el dueño del retail en estudio, así como el cargo honorífico que este tendría en Cencosud. Con esta información entregada, la fiscalía no toma ninguna decisión, pero dentro de los escenarios existentes no se descarta que solicite cumplir con algunos requisitos adicionales de llegar a concretar el negocio. (Godoy, 2011).

La misma fuente agrega que aunque no se ha cerrado el negocio, al 15 de noviembre, Cencosud se ha tomado las oficinas de la compañía en estudio, esto porque están realizando el proceso de selección de la nueva planta ejecutiva del retailer, comenzando con las entrevistas correspondientes, para determinar quiénes continuarán y quiénes se irán, además de evaluar cuáles serán los ejecutivos que se cambiarán de Cencosud a la nueva empresa.

Con esto, se comienza a dilu- cidar el resultado que tendrá el proceso, una vez terminado el Due Diligence.

\section{MES 8}

\section{- Redacción y negociación de} contratos

El día 9 de diciembre, Cencosud envía a la SVS un hecho esencial, en el cual destaca lo siguiente:

Con esta fecha, Cencosud S.A. y los accionistas de la empresa en estudio, han suscrito un memorándum de acuerdo, en el cual han convenido los principales términos y condiciones para el ingreso de Cencosud S.A. como accionista a la sociedad, por un pago total de $\$ 32.606 .000 .000$, adquiriendo en forma directa 0 indirecta una participación de $85,58 \%$ del total de las acciones de la sociedad. Junto con lo anterior, han establecido la estructura jurídica y los actos de contratos necesarios para llevarla a cabo, todo ello, sujeto a ciertas condiciones y términos habituales en estos tipos de operaciones, incluyendo el acuerdo en cada uno de los documentos definitivos.

\section{MES 9}

\section{- Cierre de la operación}

El 20 de diciembre, Cencosud S.A., pone fin a la operación de compra informando esta situación a través de un nuevo hecho esencial enviado a la SVS, en cual destaca lo siguiente:

Con esta fecha se ha suscrito un acuerdo de inversión, mediante el cual Cencosud S.A. se ha convertido en controlador de la sociedad continuadora del negocio de tiendas por departa- 
mento de la compañía en estudio. De esta manera, Cencosud S.A. es titular del $85,58 \%$ del total del capital social de dicha sociedad, aportando un monto total de \$32.606 millones. Con dichos fondos se pagaron $\$ 17.576$ millones (casi la totalidad de los pasivos bancarios), destinando el remanente al capital de trabajo de la compañía.

Además, también se destaca dentro de este hecho esencial, que la sociedad en estudio, actualmente, opera cuarenta tiendas por departamento en Chile y trece tiendas FES, con una superficie de venta de 120.00 $\mathrm{m}^{2}$. Las ventas del año 2010 alcanzaron CLP 148.000 millones.

Al mismo tiempo los medios de prensa escritos destacaban que con el cierre de la operación, Cencosud ampliaría su presencia a todo Chile, ya que la empresa en estudio opera en tiendas en ciudades donde Cencosud no está. Ciudades como Vallenar, Coquimbo, Ovalle, Los Andes, San Felipe, Quillota, Mall del Centro, San Fernando, Linares, Valdivia y Punta Arenas, igualmente podrá acceder a un segmento C3-D (clase económica media baja). (Tapia, 2011)

Dentro del proceso de Due Diligence uno de los aspectos más relevantes es que este puede traer consecuencias y producir variados efectos dentro de la organización a la cual se le realizaría. Lo anterior se debe a que uno de los aspectos primordiales, como se ha mencionado a lo largo de la investigación, es que este se realiza por el mandato de un tercero, que es el inversionista, lo que hace que este proceso sea mucho más riguroso ya que el inversionista no tiene conocimiento de lo que es la empresa en su totalidad.

De acuerdo a los criterios de Iriarte, San Sebastián, y Sainz (2010), el proceso se puede inferir de tres formas, de acuerdo a los resultados que pudiese tener. Existen criterios, que se tomarán en cuenta para relacionar los resultados obtenidos del Due Diligence de la empresa en estudio.

Los criterios son:

- Rebajar el precio de adquisición.

- Establecer garantías adicionales (por ejemplo, aval bancario, etc.).

- Fraccionar el precio.

- Desistir de la compra.

Dichos criterios expresan muy bien la finalidad del proceso de Due Diligence. A través de la investigación se ha podido establecer que una de las finalidades del proceso es que este mitigue 0 ayude a reducir riesgos para el comprador 0 inversor. Esto, evaluando de manera fehaciente la veracidad de la información y el grado de fiabilidad; las garantías de la empresa y si éstas son suficientes para el inversor. Además, se debe evaluar las posibles correcciones del precio fijado en las cláusulas del negocio, que se pactó en un principio dentro de la carta de intenciones.

De acuerdo con la investigación y a los datos obtenidos, el resultado de este proceso se puede destacar en el hecho esencial enviado el veinte de diciembre 
del 2011, en el cual se expresa claramente el precio que va a pagar por la empresa en estudio, la empresa inversora.

En este hecho esencial, que se dio a conocer anteriormente, se ve el precio real a pagar por parte de Cencosud S.A. el que pagará M\$32.606 POR EL $85,58 \%$ del capital social de la empresa.

Con este último hecho esencial y comparándolo con el primero, que es entregado el día siete de octubre de 2011, en el cual la empresa daba a conocer que había enviado una carta de intenciones a la empresa en estudio, en la que demostraba su finalidad de invertir en la empresa comprometiéndose a una participación de un 85,58\%, por un monto de $\mathrm{M} \$ 51.500$, esto sometido a una serie de condiciones, condiciones que se dan a conocer el día catorce de octubre del mismo año, en donde se destaca que se realizará un Due Diligence para revisar aspectos relevantes para la empresa inversora, tales como aspectos legales, comerciales y financieros. Este proceso duraría 45 días.

Con los antecedentes entregados y una vez revisados los datos, se puede deducir e inferir que luego de realizado el Due Diligence en la empresa en estudio, la empresa inversora no desistió de invertir en la empresa y mantuvo el porcentaje de participación que se comprometió a tener una vez suscrito el capital o sea el 85,58\%, pero si influyó en el precio que se comprometió a pagar en la carta de intenciones, rebajando este precio M\$18.894, una cifra considerable si se tiene en cuenta que gran parte del capital sería para pagar acreedores y deudas pendientes, por lo que la participación de un Due Diligence es muy importante para la empresa inversora, puesto que disminuye riesgos de inversión y, además, permite tener una visión clara de la situación del negocio a invertir.

De acuerdo a la investigación realizada, con respecto a la conceptualización de un Due Diligence, el proceso comenzó, efectivamente, una vez que se envió un acuerdo de intenciones, tal y como lo indicaron Asesores Norgestión en 2010, donde después de varias negociaciones, la empresa inversora se muestra interesada en comprar la empresa en estudio y entregaba una oferta real en dinero. Para que esta oferta se convirtiese en una inversión real, la inversora puso una serie de condiciones; una de ellas es la realización, por parte de la empresa inversora, del Due Diligence. Este proceso le entregaría a la empresa inversora una mayor seguridad de proceso y el hecho de que, realmente, el precio ofrecido es justo.

\section{Propósito del Due Diligence.}

Con los antecedentes recopilados, el propósito del Due Diligence, a realizar en la empresa en estudio, se da a conocer en la carta de intenciones, donde se indica que este se centrará en aspectos legales, comerciales $y$, sobre todo, financieros de la empresa. Esto permitirá saber al inversor que es lo que compra y determinar las cualidades del negocio a adquirir, si conviene o no adquirir esta empresa, si este negocio va a tener un retorno adecuado de la inversión y cuál es el precio justo a pagar.

\section{Clases de Due Diligence.}

Los procesos realizados por la inversora al interior de la empresa en estudio y a partir de la revisión realizada, la que se basó principalmente en una exploración de tipo financiera, la que de acuerdo a la carta de intenciones se entiende como el aspecto más relevante a revisar, por el riesgo que este aspecto traía a la in- 
versión, esto por las excesivas deudas con terceros y los créditos excesivos a clientes, las que la hicieron contraer deudas sin poder cubrirlas, por lo que el aspecto financiero fue fundamental. En este aspecto los estados financieros son relevantes, se deben revisar detalladamente sus activos y pasivos, factores críticos que el comprador debe considerar para la determinación final del precio justo.

Al aspecto financiero se suma, además, la revisión laboral, la que considera situaciones importantes desde este punto de vista, tales como juicios 0 litigios pendientes con trabajadores, indemnizaciones que se les pagarán a los trabajadores, situación de los trabajadores tales como problemas con sindicatos, beneficios 0 acuerdos que tengan los trabajadores con la empresa en estudio. Esto es relevante, ya que todas estas consideraciones tienen un costo asociado que si es elevado, puede tener riesgos importantes para la empresa inversionista. Estos costos asociados permanecen una vez concretado el negocio y, por tanto, la inversora es quién se va hacer cargo de estos gastos.

Por último, se encuentra la revisión tributaria, en la que al igual que en la revisión laboral, existen aspectos tributarios no cumplidos y, por ende, con multas, constituyéndose en costos que pueden ser muy altos.

Las tres clases de revisiones fueron indicadas, en un hecho esencial, como relevantes para la realización de la compra, ya que los costos por infringir la ley y la obtención del valor real de activos y pasivos, pueden afectar ampliamente al precio a pagar.

\section{Proceso de Due Diligence financiera.}

Dentro de la investigación realizada, se consideraron los pasos a seguir en el proceso de Due Diligence financiero, para esto se consideró un cuadro sacado directamente de investigaciones anteriores (cuadro $N^{\circ} 1$ ), en este cuadro se consideraban las etapas de desarrollo de un proceso de Due Diligence y lo que se debiese realizar, teóricamente. Con esta información teórica y teniendo antecedentes recopilados con información pública, a través de fuentes serias como Diario Estrategia, SVS, Cencosud, entre otros, se hizo una comparación entre lo que dice la teoría y lo realizado por la empresa en estudio, lo que resultó mayoritariamente coincidente con la teoría.

A través del análisis comparativo se obtuvieron resultados muy importantes para la investigación, puesto que lo expresado en la teoría se cumple mayoritariamente al momento de realizar el proceso, por lo que el orden es específico al momento de realizar el proceso.

\section{Tipos de transacciones que consid- eran realizar un Due Diligence.}

De acuerdo a los tipos de transacciones que se requiere para llevar a cabo un proceso de Due Diligence, se puede decir que el caso de la empresa en estudio es una adquisición, esto dado que la empresa adquirente pasó a tener el control de la empresa en estudio, a través de un aumento de capital. Con lo anterior, la sociedad adquirente pasa a ser matriz de la adquirida, tomando el control de sus decisiones.

\section{Fases del Due Diligence}

De acuerdo a lo investigado y con la información encontrada a través de información pública, el proceso de Due Diligence es de tipo confidencial porque las fases en que se necesita información concreta, donde se especifiquen problemas económicos reales de la empresa en estudio, no es posible 
de revelar por lo que solo se pudo mencionar los aspectos revisados en una empresa retail, desde el punto de vista de los riesgos que conlleva el tipo de negocio.

- Fase preliminar: en esta etapa se debe solicitar, de acuerdo a lo que señalan investigadores anteriores, los profesionales a utilizar, de acuerdo lo que interesa al inversor investigar con el Due Diligence de la empresa que desea adquirir. En el caso en estudio, los aspectos que más les interesan son los legales, comerciales y financieros, de acuerdo con esto PricewaterhouseCoopers, debió poner a disposición los profesionales indicados para realizar el trabajo de manera de dejar conforme a su cliente. En este caso, la empresa inversora. Teniendo esto, la empresa encargada de realizar el trabajo de Due Diligence se debe organizar, de tal manera, que pueda realizar el trabajo en el tiempo definido y, además, cumpla con las exigencias requeridas.

- Fase de investigación: de acuerdo a lo señalado por la adquirente en su memoria, lo primero que se realizó en el proceso fue la determinación del precio justo, por lo que se debió determinar el precio justo de todas las partidas de los estados financieros, para determinar una oferta preliminar, esto es, uno de los aspectos relevantes que menciona Restrepo (2008), dentro de lo señalado en la teoría, ya que, según éste esto sirve para establecer un valor de referencia. Pero en esta etapa, lo anterior no es todo lo que se debe realizar dentro de esta fase, sino que existen elementos que se deben considerar, ya que determinarán el precio final. Estos son los aspectos legales, financieros, tributarios y medioambientales. Los investigadores entregan detalles de esos aspectos de manera general para todas las empresas, pero de acuerdo a lo realizado en la investigación se consideraron los aspectos importantes que se tomarán en cuenta por el tipo de negocio de la empresa en estudio, una empresa ligada al retail, por lo que se razonaron los aspectos legales, tributarios y financieros, de manera de entregar con estos las partidas más importantes que se debiesen revisar y los riesgos que éstas traerían a la empresa en estudio.

- Fase de revisión de las operaciones: esta fase está ligada, principalmente, al giro del negocio que se está adquiriendo, por lo que en el caso de la empresa en estudio, la revisión de las operaciones estuvo enfocada a los riesgos que conlleva el negocio del retail, como la gran cartera de clientes, sus deudas, volúmenes de ventas anteriores, tamaño en el mercado y crecimiento que tendrá la inversora si compra la empresa.

- Fase de revisión de aspectos legales: dentro de esta fase, según Restrepo (2008), se debía analizar los contratos existentes, litigios pendientes 
u garantías, en la empresa en estudio. De acuerdo a lo analizado, uno de los aspectos importantes que se debe llevar a cabo en la revisión de una empresa retail se encontraban estas partidas destacadas para su revisión ya que traen consigo riesgos importantes que pueden terminar acarreando costos en dinero que afecten de manera importante los estados financieros.

- Fase de revisión de la información: según investigaciones anteriores, es en esta fase donde se realiza una interpretación de la información obtenida, de manera de poder explicarla con claridad al cliente y este pueda tomar una decisión, de acuerdo a lo realizado en la empresa en estudio. Esta fase no se presenta de manera pública, es más bien de tipo confidencial ya que no se da a conocer ningún detalle o problema encontrado dentro de la empresa en estudio, si no que solo se pude especular que los realizadores del proceso de Due Diligence encontraron problemas y situaciones importantes, una vez analizada y discutida la información recopilada. Se especula esto, ya que la oferta entregada en principio por la empresa inversora fue mucho más amplia que la oferta final, por lo que se infiere que fueron los encargados del Due Diligence los que recomendaron aquello.

- Fase de emisión de informes: este punto tiene que ver exclusivamente con la asesoría prestada por los asesores de Due Diligence, existe una estructura específica del informe, pero este es confidencial, por lo que solo se asume que se cumplió con esta estructura en el informe de Due Diligence del proceso de compra de Cencosud de la empresa en estudio. 


\section{CONCLUSIONES.}

El Due Diligence es un proceso importante dentro de la valorización de empresas, antes de realizar una fusión o una adquisición. Es un elemento determinante a la hora de llevar a cabo uno de estos procesos, dado que le permiten al inversor tener una mayor seguridad, a la hora de hacer una inversión importante.

De acuerdo a los antecedentes recopilados a través de investigaciones anteriores, se pudo determinar que el proceso tiene una forma de desarrollarse muy específica y estructurada. Por lo tanto, al conocer de que trata el proceso de Due Diligence es mucho más fácil de entender si está enfocado a una sola empresa por lo que, en resumen, es absolutamente necesario tener un conocimiento de la metodología para llegar a una conclusión específica del proceso y poder lograr una mayor razonabilidad de lo que significa realizar un Due Diligence, antes de invertir en una empresa.

Al realizar la investigación, se pudo determinar que existen cuatro resultados claros, luego de realizado el proceso de Due Diligence, los cuales son determinantes ya que pueden afectar e influir en el proceso de venta de la empresa. Entonces, resulta que el proceso es una valorización hecha a la medida del inversor, ya que es él quién manda a realizar el procedimiento y por consiguiente, es quién se encarga de determinar cuáles son las necesidades que se tienen para realizar la compra, cuáles serán sus costos y sus beneficios y, a partir de ello, determina si asumirá o no los riesgos que conlleva la operación, los cuáles se determinan en el procedimiento de Due Diligence.

En relación a lo expuesto, se puede decir que el proceso de Due Diligence tiene muchos beneficios que son en su totalidad para el inversor, ya que es quién se encarga de llevar a cabo el proceso, por lo tanto, maneja a su conveniencia éste y lo acomoda de acuerdo a lo que él necesita y a lo que está dispuesto a arriesgar, por ende, los efectos en el precio son importantes para la empresa estudiada, esto ya que es una disminución de recursos significativa, pero al tener la necesidad de inyectar recursos con urgencia, igual les resulta conveniente. Por esto, el trato es aceptado con mutuo acuerdo de las partes. Entonces, el proceso se transforma en una venta donde la necesidad toma un papel importante y fundamental, esto ya que la vendedora necesita al inversor y éste necesita crecer en su negocio y llegar a lugares que no tiene cubiertos geográficamente y a un estrato económico distinto dentro de la sociedad, lo que hace a la compra una oferta atractiva para el inversionista, por lo que se puede inferir, además, que las intenciones claras de inversión 
y un objetivo determinado hacen que el desistir de la venta, una vez realizado el proceso de Due Diligence, sea mucho más difícil 0 casi imposible.

El resultado del Due Diligence puede proporcionar una serie de circunstancias que pueden influir en la venta de una empresa, en la empresa retail estudiada el proceso fue un elemento condicionante para la valorización de la empresa, para llegar a un precio final, a una oferta concreta que sea aceptada por la vendedora y la inversora y que sea justa para ambas. La influencia tiene que ver con el poder que se asume en el proceso de Due Diligence, al estar dentro de la empresa en venta, este poder tiene que ver con encontrar diversas situaciones que pueden afectar a la inversora una vez concretado el negocio, por lo que el análisis del Due Diligence es determinante en este punto.

Por lo tanto, la valorización de la empresa a través del Due Diligence es un proceso seguro, ya que este es influyente, tiene el poder de pedir información privilegiada y confidencial dentro de la empresa que se está invirtiendo, por lo que se puede concluir que este tipo de proceso, finalmente, es muy certero. 


\section{BIBLIOGRAFÍA.}

- $\quad$ Asesores Norgestión. (2010). ¿Que es una "Due Diligence"? Barcelona, España.

- $\quad$ Calva-Mercado, Alberto//Acus Consultores, S.C. (s.f.). Resumen de razones financieras. México.

- $\quad$ Carrera, R. (Mayo de 2011). "EL PROCESO DE DILIGENCIA DEBIDA PARA LA ADQUISICIÓN DE UNA EMPRESA DE SERVICIOS DE TRANSPORTE LIVIANO $Y$ PESADO". Guatemala.

- $\quad$ Chajón, L. (2003). Due Diligence Un Enfoque Financiero. Guatemala.

- El Mercurio. (25 de marzo de 2010). Auditoría revela que Johnson's tiene US\$ 170 millones en pérdidas y complica acuerdo con los bancos. http://diario. elmercurio.com/pram/Login.aspx?SSOAut hStatus $=7 \&$ SSOTargetUrl $=h t t p \% 3 a \% 2 f \% 2$ fdiario.elmercurio.com\%2fdetalle\%2findex. asp\%3fid\%3d\%257B59f93d0a-2465-4ffeaa71-faa .

- El Mercurio. (21 de 10 de 2011). Cencosud negocia con bancos para inyectar recursos a Johnson's. Obtenido de http://www.jec.cl/articulos/?p=18748: http://www.jec.cl/articulos/?p=18748

- $\quad$ Espiñeira, Sheldon y Asociados. (2008). Boletín de Finanzas Corporativas y Recuperaciones. Venezuela.

- $\quad$ Fanárraga, C. (s.f.). EBITDA Cash flow o Flujo de caja operativo; como herramienta de la medición. Recuperado el 11 de Diciembre de 2011, de https://docs. google.com/viewer? $\mathrm{a}=\mathrm{v} \& \mathrm{q}=$ cache: $85 \mathrm{Fm}$

Z327H8J:redcontable.com/dc/ccpl/ Comite\%2520de\%2520Finanzas/Conferenc ia\%2520Viernes\%2520Financiero/EBITDA. ppt $+\& h|=e s \& p i d=b| \& s r c i d=A D$

- $\quad$ Ferrando, K. (1 de Octubre de 2011). Tres inversionistas buscan ingresar a Johnson's en aumento de capital. Obtenido de TERCERA.CL: http://diario. latercera.com/2011/10/01/01/contenido/ negocios/10-85420-9-tres-inversionistasbuscan-ingresar-a-johnsons-en-aumentode-capital.shtml
- $\quad$ Finanzas Tour. (2011). http:// www.librostonic.com/pdf/COMO-SEHACE?-UN-ANALISIS-FINANCIERO-EN-ELMARCO-DE-UNA-DUE-... Recuperado el Noviembre de 2011

- Godoy, N. (15 de 11 de 2011). FNE consulta a Johnson's sobre rol que tendría Calderón en Cencosud. Obtenido de http://www.df.cl/fne-consulta-a-johnsons-sobre-rol-que-tendria-calderon-encencosud/prontus df/2011-11-15/212423. html: $\quad$ http://www.df.cl/fne-consulta-ajohnson-s-sobre-rol-que-tendria-calderonen-cencosud/prontus df/2011-1115/212423.html

\section{$\bullet$}

Iriarte, A., San Sebastián, A., \& Sainz, A. (2010). Guía para la transmisión de empresas. Madrid: Dirección General de Política de la Pequeña y Mediana Empresa.

- La Estrategia. (6 de Septiembre de 2011). Banco de Inversiones Contacta a Cuatro Grupos por Venta de Johnson's. http://www.estrategia.cl/detalle_noticia. php?cod $=44254$.

- La Segunda. (1 de septiembre de 2011). Johnson's: Familia Calderón contrata a banco de inversiones para buscar socio. http://www.lasegunda.com/Noticias/ Economia/2011/09/677537/johnsonsfamilia-calderon-contrata-a-banco-deinversiones-para-buscar-socio .

- La Segunda. (29 de septiembre de 2011). Johnson's vive horas cruciales con la banca ad portas de junta de accionistas. La Segunda, págs. http://www.lasegunda. com/Noticias/Economia/2011/09/685195/ johnsons-vive-horas-cruciales-con-labanca-ad-portas-de-junta-de-accionistas.

- Llorens, R. (2006). La Due Diligence en las operaciones de adquisición. Barcelona, España.

- $\quad$ Melgal, M. (s.f.). Las Razones Financieras. Recuperado el 12 de Diciembre de 2011, de http://www.ecured.cu/index. php/M\%C3\%A9todo Raz\%C3\%B3n financiera

- $\quad$ Monclús, A. (2000). Disolución y Liquidación de Sociedades. Recuperado el 6 de Diciembre de 2011, de http://ciberconta. 
unizar.es/leccion/disoliqui/100.HTM

- NOTICIAS DE GIPUZKOA (8 de Enero de 2008). ¿Qué es una 'due diligence'? Obtenido de http:// www2.noticiasdegipuzkoa.com/ ediciones/2008/01/08/deportes/futbol/ d08fut51.877524.php

- Ochoa, H. (2008). LA IMPORTANCIA DEL INFORME DEL CONTADOR PÚBLICO Y AUDITOR EN LA REVISIÓN DE NEGOCIOS (DUE DILIGENCE). Guatemala.

- $\quad$ ONEtoONE, Asesores financieros, fusiones y adquisiciones. (s.f.). ONEtoONE. Recuperado el Abril de 2012, de http://www.onetoone.es/index.php/nivelesde-servicio/teaser-ciego

- $\quad$ Papa, R. (22 de Agosto de 2011). Due Diligence para fusiones y adquisiciones. El Cronista .

- $\quad$ Penno, 0. (2010). "Fusiones \& Adquisiciones: "LEGAL DUE DILIGENCE". Ensayo de Regulación Normativa de la actividad. Argentina.

- PriceWaterhauseCoopers. (2003). Introduction to Due Diligence. Medellin, Colombia.

- $\quad$ Restrepo, I. (Diciembre de 2008). DUE DILIGENCE Financiera, una forma para garantizar la toma eficiente de desiciones en la adquisición de empresas. Medellin, Colombia.

- Roca, C. (2010). EL DUE DILIGENCE EN LAS FUSIONES Y ADQUISICIONES: UN EJERCICIO IMPRESCINDIBLE. República Dominicana: HEADRICK RIZIK ALVAREZ \& FERNANDEZ 2009.

- Rodríguez, J., Gutiérrez, M., \& Peña, M. (s.f.). Impacto en la Toma de Decisiones. Recuperado el 11 de Diciembre de 2011, de http://www.monografias.com/ trabajos13/anadeef/anadeef.shtml

- $\quad$ Sánchez, I. (2004). Razones 0 índices financieros. Valencia.

- $\quad$ Soto, H. (2 de Abril de 2011).
Introducción a la interpretación de los estado financieros de una empresa. Monterrey.

- $\quad$ Tapia, M. J. (10 de 12 de 2011). la tercera.cl. Obtenido de http://diario. latercera.com/2011/12/10/01/contenido/ negocios/10-93458-9-cencosud-tomaracontrol-de-johnsons-en-enero.shtml

- Tarabini-Castellani, L. (2010). El Proceso de la Due Dilligence. FUNDACION DE ESTUDIOS BURSATILES Y FINANCIEROS , 26-27.

- $\quad$ Trujillo, C. (2008). Las razones financieras y su empleo en el análisis y la administración financiera. cuba.

- Vargas, P. (3 al 16 de abril de 2009). Capital.cl. Recuperado el 2011, de http://www.capital.cl/reportajes-yentrevistas/la-cruzada-de-johnson-s-2.html - Vega, F. (18 de octubre de 2011). Johnson's: la trastienda del rescate. Obtenido de Capital.cl: http://www.capital. cl/reportajes-y-entrevistas/iohnson-s-latrastienda-del-rescate-2.html

- Vélez, I. (2003). Guía Práctica para negociar una firma. Colombia. 